\title{
Third-generation human mitochondria-focused cDNA microarray and its bioinformatic tools for analysis of gene expression
}

\author{
Xueyan Bai1, Jun Wu1, Qiuyang Zhang1, Salvatore Alesci2, Irini Manoli², Marc R. Blackman², \\ George P. Chrousos², Allan L. Goldstein'1, Owen M. Rennert², and Yan A. Su
}

BioTechniques 42:365-375 (March 2007)

doi 10.2144/000112388

\begin{abstract}
To facilitate profiling mitochondrial transcriptomes, we developed a third-generation human mitochondria-focused cDNA microarray (hMitChip3) and its bioinformatic tools. hMitChip3 consists of the 37 mitochondrial DNA-encoded genes, 1098 nuclear DNA-encoded and mitochondria-related genes, and 225 controls, each in triplicate. The bioinformatic tools included data analysis procedures and customized database for interpretation of results. The database associated 645 molecular functions with 946 hMitChip3 genes, 612 biological processes with 930 genes, 172 cellular components with 869 genes, 107 biological chemistry pathways with 476 genes, 23 reactome events with 227 genes, 320 genetic disorders with 237 genes, and 87 drugs targets with 55 genes. To test these tools, hMitChip3 was used to compare expression profiles between human melanoma cell lines UACC903 (rapidly dividing) and UACC903(+6) (slowly dividing). Our results demonstrated internal gene-set consistency (correlation $\mathrm{R} \geq$ $0.980 \pm 0.005)$ and interexperimental reproducibility $(\mathrm{R} \geq 0.931 \pm 0.013)$. Expression patterns of 16 genes, involved in DNA, RNA, or protein biosyntheses in mitochondrial and other organelles, were consistent with the proliferation rates of both cell lines, and the patterns of 6 tested genes were verified by quantitative reverse transcription PCR (RT-PCR). Thus, hMitChip3 and its bioinformatics software provide an integrated tool for profiling mitochondria-focused gene expression.
\end{abstract}

\section{INTRODUCTION}

Mitochondria, intracellular organelles widely known as the energy factories of the cell, play fundamental roles in many metabolic pathways, such as $\beta$-oxidation, the tricarboxylic acid, and urea cycles, the synthesis of steroid hormones and heme, and calcium signaling (1). Mitochondria are the only subcellular structures possessing distinct DNA (mitochondrial DNA or mtDNA) and transcription and translation machineries (2). Yet, the vast majority of mitochondrial proteins are encoded by the nuclear DNA, synthesized by ribosomes in the cytoplasm, and imported into the organelles (3). The highly integrated cross-functionality of nuclear and mitochondrial genomes is essential for maintenance of cellular homeostasis. Defects and abnormal expression of either nuclear
DNA-encoded and/or mtDNA-encoded genes can be deleterious for human organs. While nuclear DNA mutations are rare as primary mitochondrial genetic disorders (i.e., Leigh syndrome, Friedreich's ataxia, lethal infantile cardiomyopathy, carnitine palmitoyl transferase deficiency, to name a few) $(4,5)$, abnormalities in mitochondrial structure and function are increasingly recognized in common diseases, such as obesity, diabetes, cardiomyopathy, and migraine (6-8). In addition, reactive oxygen species, an inevitable by-product of mitochondrial oxidative phosphorylation, can damage DNA and have been implicated in cancer, neurodegenerative diseases, and aging (9). Furthermore, mitochondria at the intersection of many molecular pathways are a central target of diverse pharmacological agents. Many drugs have direct effects on mitochondrial ultrastructure and function, either at the DNA level or upon targeting proteins located in the inner or outer mitochondrial membrane $(10,11)$. For example, curcumin and arsenic induce apoptosis via a mitochondria-mediated pathway $(12,13)$.

A high-throughput tool for profiling transcriptomes of the entire mitochondrion would be of great importance, as it would further improve our understanding of the mitochondria-centered physiology, pathology, pharmacology, and toxicology for better diagnosis, prevention, and treatment of disease. To provide such a tool, we previously developed a first-generation human mitochondrial-focused cDNA microarray (hMitChip1, unpublished) and a second-generation human mitochondrial-focused cDNA microarray (hMitChip2) (14). hMitChip2, which contained only 501 nuclear 


\section{Research Reports}

DNA-encoded genes, was tested and validated in human skeletal muscle cells in an attempt to better understand mitochondrial involvement in glucocorticoid-induced myopathy (15). To make a comprehensively useful tool, we developed an integrated tool including a third-generation human mitochondria-focused cDNA microarray (hMitChip3; with 37 mtDNAencoded genes and 1098 nuclear DNA-encoded and mitochondriarelated genes), computing procedures, database, and gene informatics. We tested these tools by comparing the transcriptomes of a rapidly dividing melanoma cell line UACC903 and a slowly dividing derivative cell line UACC 903(+6) $(16,17)$. Our results demonstrate that the high quality hMitChip3 and the accompanying software provide a novel integrated tool to facilitate human mitochondriaoriented research.

\section{MATERIALS AND METHODS}

\section{Gene Selection, Microarray Design, and Fabrication}

Genes listed in the Mitoproteome database (www.mitoproteome.org/ $\mathrm{html} /$ database.html) were selected for hMitChip3. In addition, the National Center for Biotechnology Information (NCBI) and other public databases were searched using keywords mitochondrial biogenesis and oxidative stress as previously described (14). Transcriptional loci predicted from sequences were not included. cDNA clones for the 37 human mtDNA-encoded genes were synthesized and sequence-verified by Geneart (Regensburg, Germany) based on GenBank ${ }^{\circledR}$ sequence (accession no. AP008773). Sequence-verified cDNA clones for nuclear DNAencoded genes were purchased from Invitrogen (Carlsbad, CA, USA) and Origene (Rockville, MD, USA). Gene information was updated based on Unigene Build 189 (www.ncbi.nlm. nih.gov/UniGene). Test genes (1135), 146 positive controls (housekeeping and duplicate genes), and 79 negative controls (print-buffer without DNA) were printed in triplicate onto each hMitChip3 slide (see Supplementary
Table S1 available online at www. gwumc.edu/biochem/faculty/su.html) as previously described (18), using the OmniGrid ${ }^{\circledR} 100$ microarrayer (Genomic Solutions, Ann Arbor,
MI, USA). Spike-in DNA controls for possible contamination (plasmid DNA), blocking efficiency (salmon sperm DNA), and cross-hybridization (human Cot-1 repetitive DNA), previ-

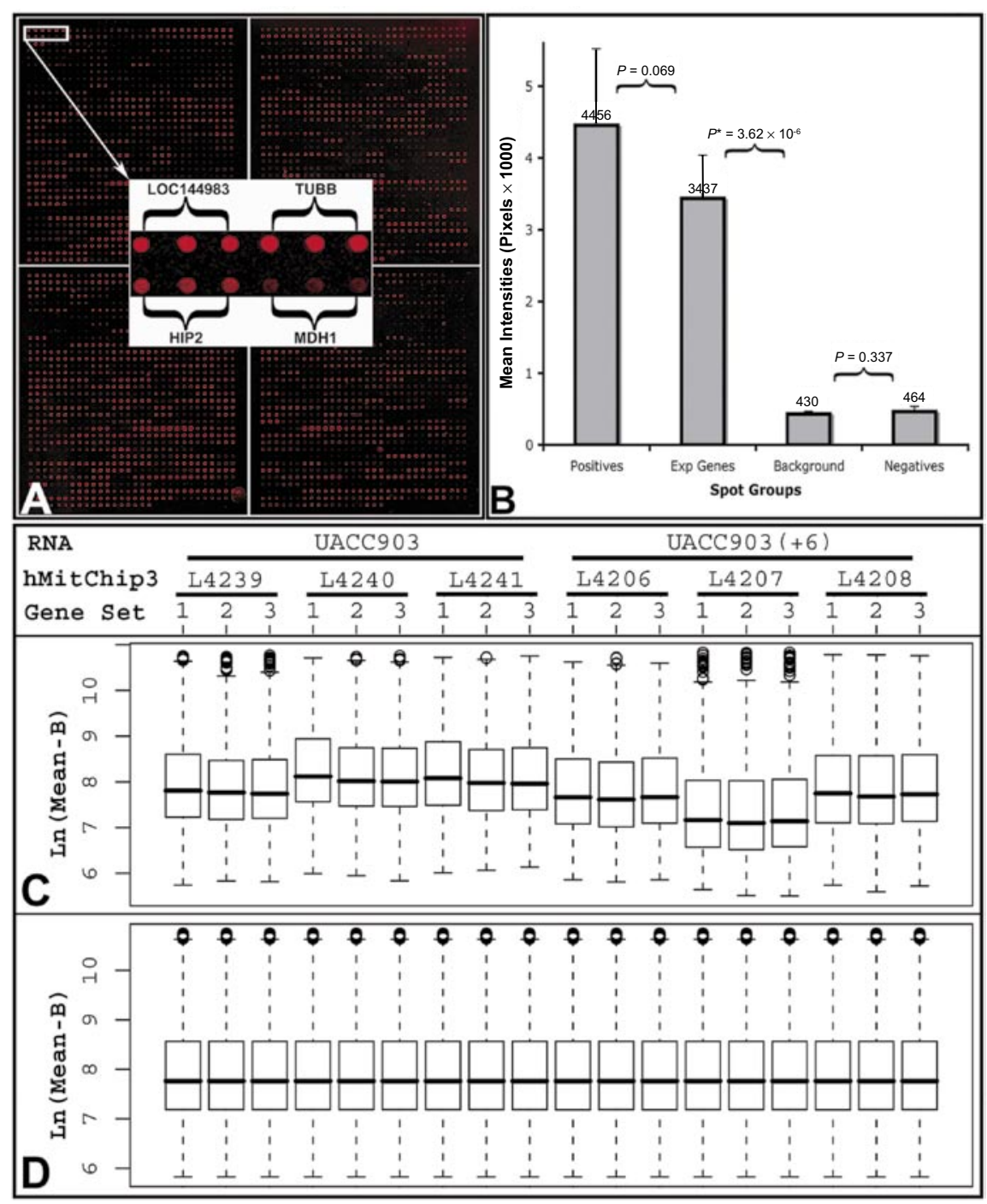

Figure 1. hMitChip3 representative image, quality, and data normalization. (A) Representative microarray image. This pseudocolored image represents a hMitChip3 microarray hybridized with the Cy5labeled target cDNA reverse-transcribed from a UACC903 RNA sample. Four printing heads were used to print four subarrays of the image, and each element was printed as triplicate (with triplicates adjacent to each other). The pixel intensities on spotted probes reflect abundances of hybridized target cDNA. Inset shows four genes with high (LOC144983 and TUBB), moderate (HIP2), and low (MDH1) signal intensities. (B) Bar graph illustrates mean pixel intensities of the positive $(n=438)$ and negative $(n=$ $237)$ control spots, test gene spots $(n=3405)$, and the background of all the spots $(n=4080)$. The mean and standard deviation of the positive, test gene, negative, and background were $4456 \pm 1068,3437 \pm$ $602,464 \pm 75$, and $430 \pm 41$, respectively. $P$ values $(P)$ between comparisons are indicated. $P^{*}$ means statistically significant $P$ value $\left(3.62 \times 10^{-6}<0.05\right)$. LOC144983, heterogeneous nuclear ribonucleoprotein A1-like; TUBB, $\beta$-tubulin; HIP2, Huntingtin-interacting protein 2; $M D H 1$, malate dehydrogenase 1. (C and D) Boxplots before and after data normalization. The quantile normalization algorithms (20) were used to adjust the natural log of the background-subtracted mean pixel intensities of each and every set of 577 genes that were selected from the hMitChip3 triplicate microarray experiments by the criteria described in the text. In contrast to (C) the prenormalization boxplots, (D) the postnormalized boxplots distribute in the same intervals with the same density center, indicating a successful location adjustment. The postnormalized data were used for further analysis to identify differentially expressed genes with statistical significance $(P$ value $<0.05)$. 


\section{Research Reports}

ously included in hMitChip2, were not necessarily repeated here $(14,15)$.

\section{Cell Cultures}

Human melanoma cell lines UACC 903 and UACC $903(+6)$ were originally obtained from the University of Arizona Cancer Center (16). UACC903 was cultured in RPMI 1640 supplemented with $10 \%$ fetal bovine serum, $2 \mathrm{mM}$ L-glutamine, $100 \mathrm{U} / \mathrm{mL}$ penicillin G sodium, and $100 \mu \mathrm{g} / \mathrm{mL}$ streptomycin sulfate. UACC $903(+6)$ was cultured in the same medium after addition of $600 \mu \mathrm{g} / \mathrm{mL}$ G418 to select for the pSV2neo-tagged chromosome 6 (16). Media, sera, and antibiotics were purchased from the GIBCO cell culture (Invitrogen). RNA samples for triplicate experiments were extracted from each cell line at the same passage cultured under the same conditions.

\section{Microarray Hybridization and Image Scanning}

Total RNA was extracted using TRIzoL ${ }^{\circledR}$ reagent (Invitrogen) and purified with RNeasy ${ }^{\circledR}$ kit (Qiagen, Valencia, CA), as described previously (15). Ten micrograms RNA per sample were used for Cy ${ }^{\mathrm{TM}} 5$-dUTP labeling of cDNA and microarray hybridization using methods previously described (15). Slides were scanned using the ScanArray ${ }^{\circledR}$ Express microarray scanner (PerkinElmer, Wellesley, MA, USA) under $90 \%$ laser power, 68 photomultiplier tube (PMT) voltages, 5 $\mu \mathrm{m}$ resolution, and LOWESS method. Each scanned spot was labeled either as 0 (found but not good), 1 (not found), 2 (absent), 3 (good), or 4 (bad). The good spots were defined by the scanner software setups as the spot with a calculated footprint $<100 \mu \mathrm{m}$.

\section{Database Compilation}

A database was compiled in FileMaker Pro (FileMaker, Santa Clara, CA, USA) and includes individual and relational expression files and relational and category gene information files. Individual expression files were built for quantitative text files derived from scanned images with all names matching exactly those of the text files. The relational expression file links all individual expression files via the index (i.e., the unique numerical identifier for each spot) and contains self-explanatory information for data filtering and comparisons. The relational gene information file contains expression summary and gene biological information. Each category gene information file organizes gene bioinformation into self-explanatory groups.

\section{Data Analysis}

Scatter plots, fitting lines, and the Pearson correlation coefficient $(R)$ were calculated using Microsoft $^{\circledR}$ Excel $^{\circledR}$. Microarray data were filtered within hMitChip3 database using the following criteria: $(i)$ test gene spot $=$ yes; $(i i)$ spot flag $=3$ (good spots); (iii) signal-to-noise ratio (i.e., spot mean pixel intensity minus background mean pixel intensity and then divided by background standard deviation) $\geq 2$; (iv) saturation of spot pixel intensities $\leq 75 \%$ (i.e., approximately linear quantitative intensities); and $(v)$ $\geq 25 \%$ pixel intensities of a spot above 2 standard deviations higher than background mean intensity (i.e., good spot morphology). The backgroundsubtracted mean intensities of the selected data were normalized across all intra- and interslide spots and array experiments. The normalized data were used to calculate average intensity, ratio, standard deviation, and $P$ value.

\section{Gene Bioinformation}

Gene identifiers, including IMAGE clone ID, GenBank accession no., Unigene ID, gene ID, symbol, and name, were downloaded from the NCBI Human Unigene Database (ftp. ncbi.nih.gov/repository/UniGene). Gene ontology (molecular function,

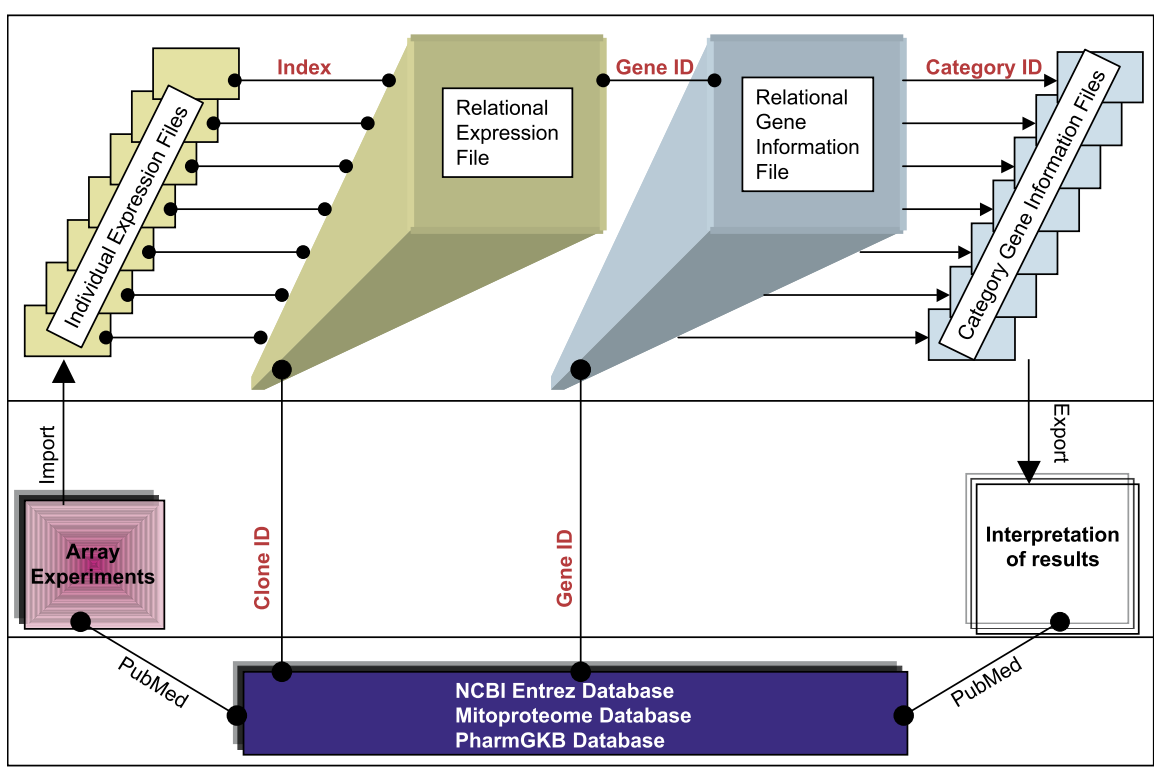

Figure 2. Overview of hMitChip3 database structure. hMitChip3 database includes individual expression files, a relational expression file, a relational gene information file, and category gene information files. Each individual expression file keeps raw microarray data imported from image scanning of a microarray hybridization based on design of array experiments and is linked with the relational expression file via index (a unique numerical ID to each spot on hMitChip3) (see Figure 3A). The relational expression file contains information on array design, expression data and comparison, and some gene information (see Figure 3B). The relational gene information file contains results of expression comparisons and gene biological information (see Figure 4A) and is linked with the relational expression file via the NCBI Entrez Gene ID. All or partial information in the relational gene information file can be exported into category gene information files (see Figure 4B) for category analysis and interpretation of results. A design of microarray experiments, analysis of data, and interpretation of results need information from public databases. Arrowheads indicate the direction of information flow. Lines with dots at two ends indicate links. 


\section{Research Reports}

biological process, and cellular component), phenotype (genetic disorder), and pathways (KEGG pathway and reactome event) were downloaded from the Entrez Gene Database (ftp.ncbi.nlm.nih.gov/gene). Drug targets associated with gene products were from PharmGKB (www. pharmgkb.org/search/index.jsp).

\section{Quantitative Reverse Transcription PCR}

Two micrograms total RNA samples were reverse-transcribed into cDNA by using SuperScript ${ }^{\mathrm{TM}}$ First-Strand Synthesis System (Invitrogen). The real-time PCRs were performed using TaqMan ${ }^{\circledR}$ Universal PCR Master Mix (Applied Biosystems, Foster City, CA, USA), following the manufacturers' instructions. Relative RNA concentrations were calculated using the published methods $(15,19)$. TaqMan probes and primers for human glyceraldehyde-3-phosphate dehydrogenase $(G A P D H)$, histone deacetylase 3 (HDAC3), DNA (cytosine-5-)-methyltransferase 1 (DNMT1), chaperonin containing TCP1 (CCT4), nonmetastatic cell 4 (NME4), GA binding protein transcription factor (GABPA), and peroxisome proliferative activated receptor $(P P A R G)$ were purchased from Applied Biosystems. Triplicate quantitative reverse transcription PCR (RT-PCR) experiments were performed for each gene.

\section{Statistics}

Statistical calculations were performed on triplicate array experiments using XLSTAT 2006 (XLSTAT, New York, NY, USA). The quantile normalization described by Bolstad et al. (20) was carried out using $R$ 2.2.1 software (The R Foundation for Statistical Computing, Vienna, Austria). As a minimum, a 2-fold difference in the background-subtracted mean intensity ratio of a gene between samples and controls was used as an indicator of differentially expressed genes. Student's $t$-tests were used to calculate $P$ values for the ratio difference. The level of statistical significance was set at a $P$ value $<0.05$.
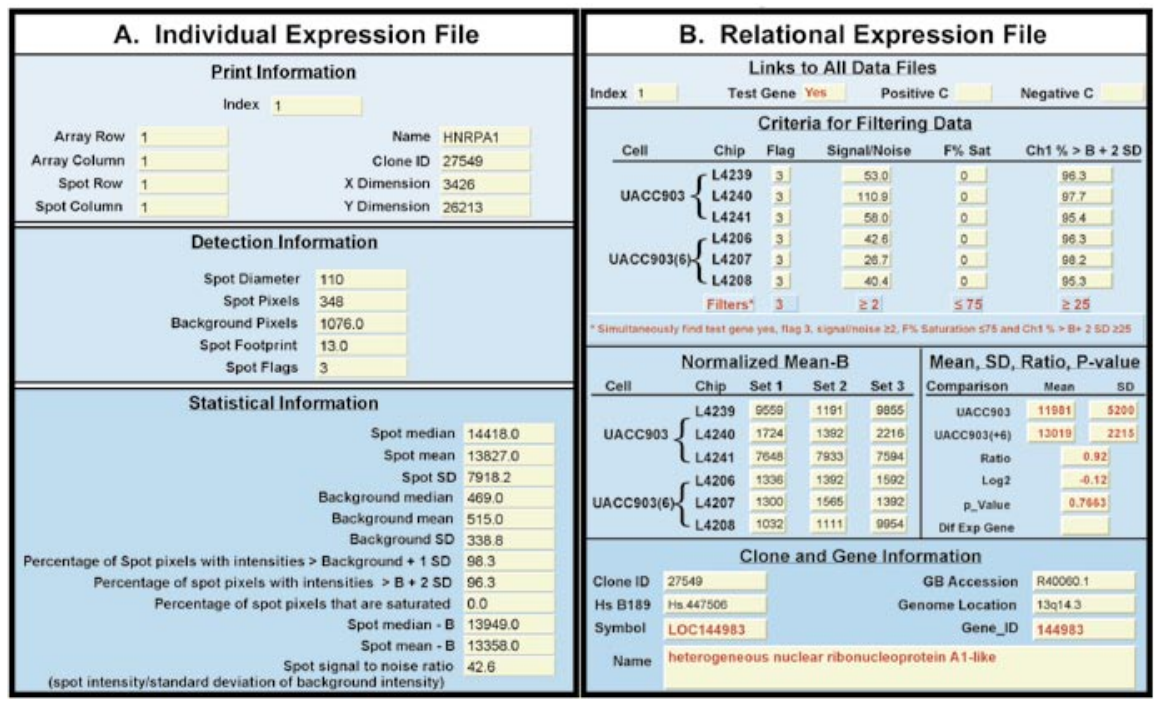

Figure 3. User interface-1 of hMitChip3 database compiled in FileMaker Pro. (A) Individual expression file allows storage and display of the print information, detection information, and statistical information imported directly from output of scanned Excel text file. All names listed are the same as those of the text file. (B) Relational expression file links all individual expression files via the index (i.e., the unique numerical identifier for each spot) and contains self-explanatory information for data filtering and comparisons. The databases allow searching, browsing, displaying, and modifying, according to experimental designs. Templates of hMitChip3 database with self-explanatory information can be downloaded at www.gwumc.edu/biochem/faculty/su.html.

\section{RESULTS}

\section{Uniformly Low Background of the hMitChip3 Gene Chips}

The quality of hMitChip3 slides was assessed in triplicate microarray experiments using RNA samples derived from human melanoma cell lines UACC903 and UACC903(+6). Results showed uniformly low background intensity on all hybridized chips, with a distribution of signal intensities from 65,535 pixels $(<1 \%$ of 4080 spots) gradually down to the background (Figure 1A). Specifically, the averages of the mean pixel intensities for the positive, test, background, and negative spots (see Supplementary Table S1), calculated from all the six hybridized chips (regardless of the RNA sample used), were $4456 \pm 1067,3437 \pm 602$, $430 \pm 41$, and $464 \pm 75$, respectively (Figure 1B). On average, the signal intensities of the test spots (3437 pixels) were 8 -fold higher than those of the background (430 pixels), and this difference was highly significant $(P=$ $\left.3.62 \times 10^{-6}\right)$. In contrast, the difference between test and positive spots was not statistically significant $(P=0.069)$.
There was essentially no difference in mean intensities between negative spots and background $(P=0.337)$ (Figure 1B).

\section{High Signal-to-Noise Ratio and Equal Overall Spot Pixel Intensities}

Table 1 lists the signal-to-noise ratios and averages of the backgroundsubtracted mean pixel intensities. On average, the signal-to-noise ratio was 12.6-fold for the test gene spots and 16.6- and 1.4-fold for the positive and the negative controls, respectively. The corresponding spot backgroundsubtracted mean pixel intensities were 3064, 4079, and 93, respectively. The difference in average signal-tonoise ratios between UACC903 and UACC903(+6) cDNA hybridized chips were $1.27,1.42$, and 0.82 for test genes, positive, and negative controls, respectively. The corresponding difference in the spot background-subtracted mean pixel intensities between the two cell lines was $1.30,1.38$, and 1.07 , respectively. These differences were not statistically significant (Table 1), although the average signal-to-noise ratios and spot background-subtracted mean pixel 


\section{Research Reports}

intensities of the test genes and positive controls for UACC903 were slightly higher than those for UACC903(+6). Therefore, high signal-to-noise ratio and equal overall pixel intensities for UACC903 and UACC903(+6) samples were obtained using hMitChip3.

\section{Similarity Between the Identical Gene-Set Intensities}

To compare intensities among identical gene-sets measured by hMitChip3, we conducted $2 \times 2$ comparisons of the spot backgroundsubtracted mean pixel intensity for the test genes and positive controls on six hybridized chips and calculated Pearson correlation coefficients $(R)$ for the Proximity Matrix (Table $2)$. The matrix showed intensity similarities $(R)$ for each set of genes, both within the same chip and between different chips, as well as between RNA samples obtained from the two different cell lines. The intra-hMitChip3 values of the mean similarity and standard deviation were $0.980 \pm 0.005$ and $0.981 \pm 0.011$ for UACC903 and UACC903(+6), respectively. The corresponding interhMitChip3 values were $0.931 \pm 0.013$ and $0.944 \pm 0.017$, respectively. The mean similarity and standard deviation between UACC903 and UACC903(+6) samples were $0.879 \pm 0.020$, which as expected was the least among all the correlation coefficients (Table 2).

\section{Computing Procedures for Identification of Differentially Expressed Genes}

To identify differentially expressed genes, we customized computational procedures including data evaluation, filtering, inclusion, normalization, and comparison. Data evaluation is aimed at revealing data quality including background, signal-to-noise ratio, overall spot pixel intensity, and consistency. Data filtering is aimed at removing (with uniform criteria described in the Materials and Methods section) all spots that may lead to false positives or negatives. Data inclusion is aimed at determining whether all spots fulfilling the criteria for data filtering should be used for further analysis. Because some of the 9 spots/gene (i.e., triplicate spots/chip from triplicate experiments) may not fulfill the filtering criteria, some of the filtered genes across all array experiments may have values from $<9$ spots. Alternatively, one may include only genes with all the 9 spots fulfilling the filtering criteria for further analysis. The former complicates data analysis because of missing values. The latter gave a subset of genes with no missing data; however, the number of genes included in the final result may be reduced. In the current experiments, 2029 of 3405 test gene spots satisfied the filtering criteria. Of these 2029 spots, $1731(85 \%)$ represented the 577 genes for which all the 18 spots/ gene (i.e., triplicate spots/chip, from triplicate array experiments performed in two cell lines) met the criteria; 240 (12\%) spots represented the 120 genes for which 12 out of 18 spots/gene and 6 spots/cell line passed the filtering criteria; and 58 (3\%) spots represented the 58 genes with 6 spots/gene and 3 spots/cell line that passed the filtering criteria. In this analysis, only the 577 genes were used for further analysis.

Data normalization is aimed at removing nonbiological variations that can arise in any steps of microarray experiments, including array printing, RNA preparation, labeling, hybridization, or scanning (21). Before and after performing quantile normalization (20), the background-subtracted mean pixel intensities of 10,386 spots (i.e., triplicate spots/gene/chip, of 577 genes included, from triplicate array experiments, performed on two cell lines) were transformed to natural $\log$. The $\log$-transformed values were plotted as the boxplots to visualize successful adjustment of the data by graphical comparisons (Figure 1, C and D).

Data comparison is to compute averages, standard deviations, ratios, and $P$ values of the normalized spot background-subtracted mean pixel intensities between desired comparisons in order to reveal differentially expressed genes. An intra- and interhMitChip3 average of triplicate genesets and triplicate experiments should reduce variance in pixel intensities. Ratios of averages between test and reference samples provide comparisons of transcriptional profiles. $P$ values are calculated from triplicate array experiments $(n=3)$ (i.e., 3 averages of triplicate gene-sets/array experiment for each cell line). When using 2-fold difference as threshold, we identified 66 differentially expressed genes between UACC903 and UACC903(+6) cells (Supplementary Table S2).

\section{hMitChip3 Database}

To expedite data analysis and interpretation of results, we built the hMitChip3 database for data organization, computation, interpretation, and visualization. Figure 2 is a diagrammatical overview of the hMitChip3 database. The individual expression files are used to store microarray data, including printing, detection, and statistical information (Figure 3A). The relational expression file links all the individual expression files and provides user-interface to allow importing, searching, browsing, computing, comparing, displaying, and exporting of data (Figure 3B). Containing gene bioinformation, the relational gene information file (Figure 4A) provides user-interface to allow importing, searching, browsing, displaying, and exporting of $(i)$ genes of interest, with the associated expression changes and bioinformation; (ii) expression changes of interest, with the associated genes and bioinformation; and (iii) bioinformation of interest, with the associated genes and expression results. The category gene information files consist of individual files of molecular function, biological process, cellular component, KEGG pathway, reactome event, genetic disorder, and PharmGKB drug targets. Because of multiple genes under each bioinformation and multiple bioinformation under each gene, and their association with expression changes, information in each category file can be organized by sorting based on genes, expression, or bioinformation of interest. As an example, Figure 4B shows partial biological process focused on antiapoptosis. Both relational and category gene information files are linked to public databases via the NCBI Entrez Gene ID. Templates of hMitChip3 database with self-explanatory infor- 


\section{Research Reports}

mation can be downloaded at www. gwumc.edu/biochem/faculty/su.html.

\section{hMitChip3 Gene Bioinformation}

Bioinformation of $1135 \mathrm{hMitChip} 3$ test genes were compiled and imported into relational and category gene information files to facilitate the biological interpretation of differentially expressed genes. Specifically, we were able to link 946 hMitChip3 genes with 645 molecular functions (Supplementary Table S3), 930 genes with 612 biological processes (Supplementary Table S4), 869 genes with 172 cellular components (Supplementary Table S5), 476 genes with 107 KEGG biological chemistry pathways (Supplementary Table S6), 227 genes with 23 reactome events (Supplementary Table S7), 237 genes with 320 genetic disorders (Supplementary Table S8), and 55 genes with 87 PharmGKB drug targets (Supplementary Table S9). All supplementary tables are available online at www.gwumc.edu/biochem/faculty/su. html.

\section{Quantitative RT-PCR Verification of Differentially Expressed Genes}

Applying the hMitChip3 gene information to 66 differentially expressed genes (Supplementary Table 2), we found 16 genes with known functions involved in cell cycle progression and DNA, RNA, or protein biosynthesis (Table 3) for mitochondrial and other organelles. The reason focusing on these functions is that a growth rate of UACC 903 cell line is twice as rapid as that of UACC903(+6) cell line (17). Our quantitative RT-PCR results on six tested genes demonstrated agreement with the microarray results (Table 3). Specifically, the complete consistent expression changes include $H D A C 3$ (2.96-fold, $P=0.0386$ ), DNMT1 (1.69-fold, $P=0.0287$ ), and NME4 (1.64-fold, $P=0.0327$ ) with higher expression and PPARG $(0.18$-fold, $P$ $=0.00000004)$ with lower expression in UACC903 than in UACC903(+6). CCT4 and GABPA were up-regulated by 1.81 -fold $(P=0.2638)$ and 2.03 fold $(P=0.2626)$ higher, respectively, in UACC903 than UACC903(+6);

Table 1. S/N Ratio and Average Intensitives of hMitChip3 Spots

\begin{tabular}{|c|c|c|c|c|c|c|c|c|c|}
\hline \multirow{2}{*}{ 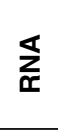 } & \multirow{2}{*}{ 은 } & \multicolumn{2}{|c|}{ Spot } & \multicolumn{2}{|c|}{ Average } & \multicolumn{4}{|c|}{ UACC903 vs. UACC903(+6) } \\
\hline & & Group & $n$ & S/N & $\begin{array}{c}\text { S-B } \\
\text { Mean }\end{array}$ & $\mathbf{S} / \mathbf{N}$ & $\begin{array}{c}P \\
\text { Value }\end{array}$ & $\begin{array}{c}\text { S-B } \\
\text { Mean }\end{array}$ & $\begin{array}{c}P \\
\text { Value }\end{array}$ \\
\hline \multirow{9}{*}{$\begin{array}{l}\text { రి } \\
\text { ర্ } \\
\text { ঠ্ড }\end{array}$} & \multirow{3}{*}{ 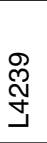 } & Test & 3405 & 12.5 & 3076 & & & & \\
\hline & & Positive & 438 & 14.5 & 3653 & & & & \\
\hline & & Negative & 237 & 1.2 & 104 & & & & \\
\hline & \multirow{3}{*}{ 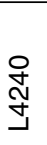 } & Test & 3405 & 15.3 & 3756 & & & & \\
\hline & & Positive & 438 & 23.4 & 5455 & & & & \\
\hline & & Negative & 237 & 1.2 & 75 & & & & \\
\hline & \multirow{3}{*}{ 六 } & Test & 3405 & 14.4 & 3577 & & & & \\
\hline & & Positive & 438 & 20.5 & 5064 & & & & \\
\hline & & Negative & 237 & 1.5 & 109 & & & & \\
\hline \multirow{9}{*}{ 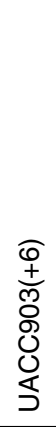 } & \multirow{3}{*}{ 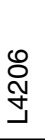 } & Test & 3405 & 11.5 & 2856 & & & & \\
\hline & & Positive & 438 & 14.3 & 3757 & & & & \\
\hline & & Negative & 237 & 1.5 & 50 & & & & \\
\hline & \multirow{3}{*}{ 突 } & Test & 3405 & 9.3 & 2064 & & & & \\
\hline & & Positive & 438 & 9.5 & 2430 & & & & \\
\hline & & Negative & 237 & 1.3 & 40 & & & & \\
\hline & \multirow{3}{*}{ 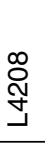 } & Test & 3405 & 12.5 & 3057 & & & & \\
\hline & & Positive & 438 & 17.2 & 4115 & & & & \\
\hline & & Negative & 237 & 2.0 & 179 & & & & \\
\hline & \multicolumn{3}{|c|}{ Test } & 12.6 & 3064 & 1.27 & 0.08 & 1.30 & 0.09 \\
\hline & \multicolumn{3}{|c|}{ Positive } & 16.6 & 4079 & 1.42 & 0.17 & 1.38 & 0.16 \\
\hline & \multicolumn{3}{|c|}{ Negative } & 1.4 & 93 & 0.82 & 0.29 & 1.07 & 0.89 \\
\hline
\end{tabular}

however, their $P$ values, in contrast to microarray results, were $>0.05$.

\section{DISCUSSION}

Gene expression profiling by DNA microarrays allows high-throughput screening for genes and pathways with key functions in diverse physiologic, pathologic, pharmacologic, and toxicologic processes. Mitochondrial research has influenced evolving concepts in evolution, physiology, pathology, pharmacology, and toxicology. An increasingly better understanding of mitochondrial disorders and the association of mitochondrial impairment with a constantly expanding list of common diseases have attracted the attention of the scientific and medical communities $(1,22)$. Accordingly, content in the human mitochondrial genome database MITOMAP has grown rapidly (23). A Human Mitochip was developed for a high-throughput screening of mutations in the mitochondrial genome (24). In addition, a cDNA microarray containing 618 genes was used to study human mitochondrial complex 1 deficiency (25), and generic cDNA microarrays and oligonucleotide gene chips have also been utilized to study mitochondrial dysfunction (26).

We previously developed and utilized hMitChip2 to study the mitochondrial involvement in glucocorticoid-induced myopathy $(15,27)$. Because hMitChip2 contained no mtDNA-encoded genes and only 501 nuclear DNA-encoded genes (14), we improved the microarray by developing hMitChip3. To our knowledge, hMitChip3 is the first customized human cDNA microarray containing all the 37 mtDNA-encoded genes and 1098 nuclear DNA-encoded and mitochondria-related genes, allowing intergenomic studies of the mitochondrial genes. The advantages 


\section{Research Reports}

of a focused microarray over human genome microarray with estimated 30,000 genes include hypothesisdriven experiments, affordable costs, simplified data analysis, and defined biological interpretation of results. However, considering that the sequence-predicted DNA loci were not included in hMitChip3 and that the number of the estimated mitochondrial genes (26) and proteins (28) are constantly increasing, a disadvantage of hMitChip3 in contrast to the whole genome gene chip probably is absence of genes that might be important to mitochondrial function.

We achieved high quality and consistency for hMitChip3 by using the highest standards in the choice of manufacturing sources and materials, design, development, and quality control. The test, positive, and negative controls were printed in triplicate on each slide to reduce random variance. For both positive controls and test genes, $>13$-fold signal-to-noise ratio was consistently reached when using $10-\mu \mathrm{g}$ RNA for labeling and hybridization, whereas the ratios of the negative controls were, as expected, around 1 . The uniformly low background $(<500$ pixel intensities) did not change when using $50-\mu \mathrm{g}$ total RNA, and in this case, the average signal-to-noise ratio ranged as high as 70-fold (14). Although using more RNA will increase the signal-tonoise ratio and lead to detection of rare RNA species, it also increased signal saturation of abundant RNA species (14). Therefore, one should adjust the amount of RNA based on an experimental design. Importantly, the equal amount of RNA samples labeled and hybridized to the spotted DNA probes on each hMitChip3 were authentically translated into the overall equal spot pixel intensities. The overall equality is essential to the identification of differentially expressed individual genes.

Nonbiological variations interfere with true biological variations and may cause false positives and negatives of differentially expressed genes. Nonbiological variations can arise in any step, including printing arrays, RNA sample preparation, labeling, hybridization, or image scanning (21). Technical variations can be removed by filters $(29,30)$. For example, spots below a detection threshold for the amount of RNA used ought to be removed for obvious reasons. In addition, spots with low intensities, lack of linear quantitation, or irregular morphology should also be excluded for lack of reliability. The hMitChip3 database simplifies the data filtering procedures into a single step: that is, to simultaneously find the flag 3 , the signal-to-noise ratio $\geq 2$, the percentage of spot saturation $\leq 75 \%$, and the spot pixel intensity $>2$ standard deviations of background $\geq 25$, across all test genes in all array experiments.

Nonbiological variations may be mixed with meaningful biological signals in the selected spots and require more sophisticated methods to decipher. To date, a number of algorithms to normalize data from various array platforms have been developed to accomplish such a daunting task (20,31-34). Because there is no gold standard to identify a best normalization method for any array platform, we decided to utilize the quantile algorithm to normalize the hMitChip3 data, partially because of the single-fluorescent (Cy5) labeling and partially because of the simplicity of this algorithm (20). It should be noted that while the positive and negative controls on hMitChip3 provide good indicators for high, medium, low, and background intensities, they are not immune to nonbiological variations.

DNA microarrays have used either one- or two-fluorescent approaches to quantify RNA in cell and tissue samples. The single-fluorescent approach is to synthesize Cy3- or Cy5labeled cDNAs for all RNA samples, hybridize to arrayed probes, measure the background-subtracted intensity at each probe on microarrays, and calculate the ratio of gene expression. The two-fluorescent approach is to synthesize $\mathrm{Cy} 3$ - and Cy5-labeled test and reference cDNA samples individually and co-hybridize them to arrayed probes, and directly measure the ratio of the background-subtracted intensities at each probe. The pros and cons of both approaches are well-known $(35,36)$. For the single-

\begin{tabular}{|c|c|c|c|c|c|}
\hline \multicolumn{3}{|c|}{ A. Relational Gene Information File } & \multicolumn{3}{|c|}{ B. Category Gene Information File } \\
\hline \multirow{2}{*}{\multicolumn{3}{|c|}{ Symbol HDAC3 }} & \multicolumn{3}{|c|}{$\begin{array}{l}\text { Biological Process } \\
\text { Gene ID Symbol Name }\end{array}$} \\
\hline & & & anterograde exor & carpo trant & \\
\hline \multicolumn{3}{|l|}{ Name histone deacetylase 3} & $\operatorname{lng}_{\text {nops }}$ & Noris & Inetin temav menter is \\
\hline & $\begin{array}{l}301 \\
300\end{array}$ & $\begin{array}{l}\text { Nochi: } \\
\text { Nowas }\end{array}$ & $\operatorname{mexinAt}$ \\
\hline & & & 8509 & NPB & noveresia metotors \\
\hline \multirow{3}{*}{\multicolumn{3}{|c|}{\begin{tabular}{rl|l|l} 
UACC903 & 3 & 4528 & 560 \\
UACC903(-6) & 3 & 681 & 263 \\
\end{tabular}}} & 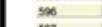 & & Bcesc Cunmotoma 2 \\
\hline & & & $\$ 97$ & BCLAAT & BCLaredaled wotein AS \\
\hline & & & ${ }_{10017}$ & scaxt & BCL2the I \\
\hline \multicolumn{3}{|c|}{ NCBI Entrez Gene Summary } & 662 & ser & 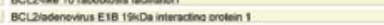 \\
\hline \multirow{7}{*}{\multicolumn{3}{|c|}{ 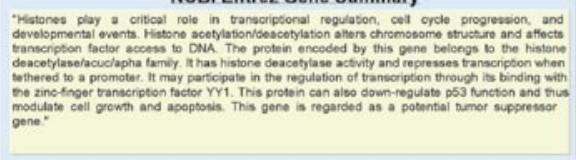 }} & 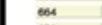 & $=\infty$ & 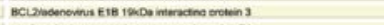 \\
\hline & & & on & w & 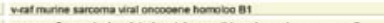 \\
\hline & & & sas & cosph & 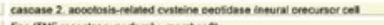 \\
\hline & & & sis & rones & 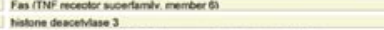 \\
\hline & & & 2000 & ispens & 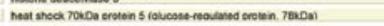 \\
\hline & & & 2313 & nspers & 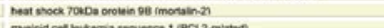 \\
\hline & & & 4770 & 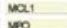 & 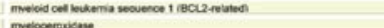 \\
\hline \multirow{2}{*}{\multicolumn{3}{|c|}{ NCBI Entrez and PharmGKB Gene Information }} & 7001 & Prose & 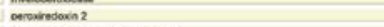 \\
\hline & & & seas & pert & 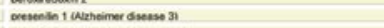 \\
\hline \multicolumn{3}{|c|}{ Molecular Funetion histone deacetylase activity } & 7420 & me & 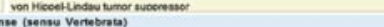 \\
\hline \multicolumn{3}{|c|}{ 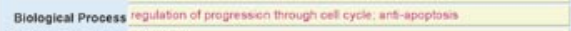 } & & & 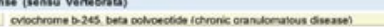 \\
\hline \multicolumn{3}{|l|}{ Cellular Component Oytoplasm } & $8 m 2$ & FADO & 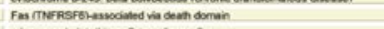 \\
\hline \multirow{2}{*}{\multicolumn{3}{|c|}{ KEGG Pathway Description }} & 2050 & mosts & 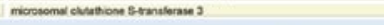 \\
\hline & & & 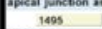 & $\begin{array}{c}\text { aemoliy } \\
\text { cranus }\end{array}$ & 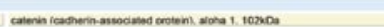 \\
\hline \multicolumn{3}{|l|}{ Reactome Event } & & & \\
\hline \multicolumn{3}{|l|}{ Cenettic Disorder } & 50 & NPS & 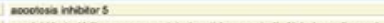 \\
\hline \multirow{2}{*}{\multicolumn{3}{|c|}{ PharmokB Drug }} & $\begin{array}{c}301 \\
\text { sot }\end{array}$ & Nep & 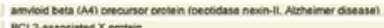 \\
\hline & & & & & 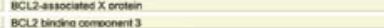 \\
\hline \multirow{2}{*}{\multicolumn{2}{|c|}{ 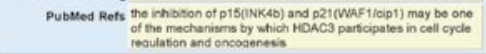 }} & 10260393 & 10134 & scapsi. & 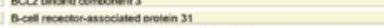 \\
\hline & & & & ave 1 & 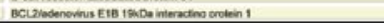 \\
\hline
\end{tabular}

Figure 4. User interface-2 of hMitChip3 database compiled in FileMaker Pro. (A) Relational gene information file contains gene expression summary and biological information as listed. All gene IDs are hyperlinked to the NCBI Entrez Gene web site for instant checking or updating information. (B) An example of category gene information file-Biological Process. Seven category gene information files include molecular function, biological process, cellular component, KEGG pathway, reactome event, genetic disorder, and PharmGKB drug targets. Each category gene information file is to organize gene bioinformation into selfexplanatory functional clusters to facilitate interpretation of gene expression profile results. The databases allow searching, browsing, displaying, modifying, and updating information. Templates of hMitChip3 database with self-explanatory information can be downloaded at www.gwumc.edu/ biochem/faculty/su.html. 


\section{Research Reports}

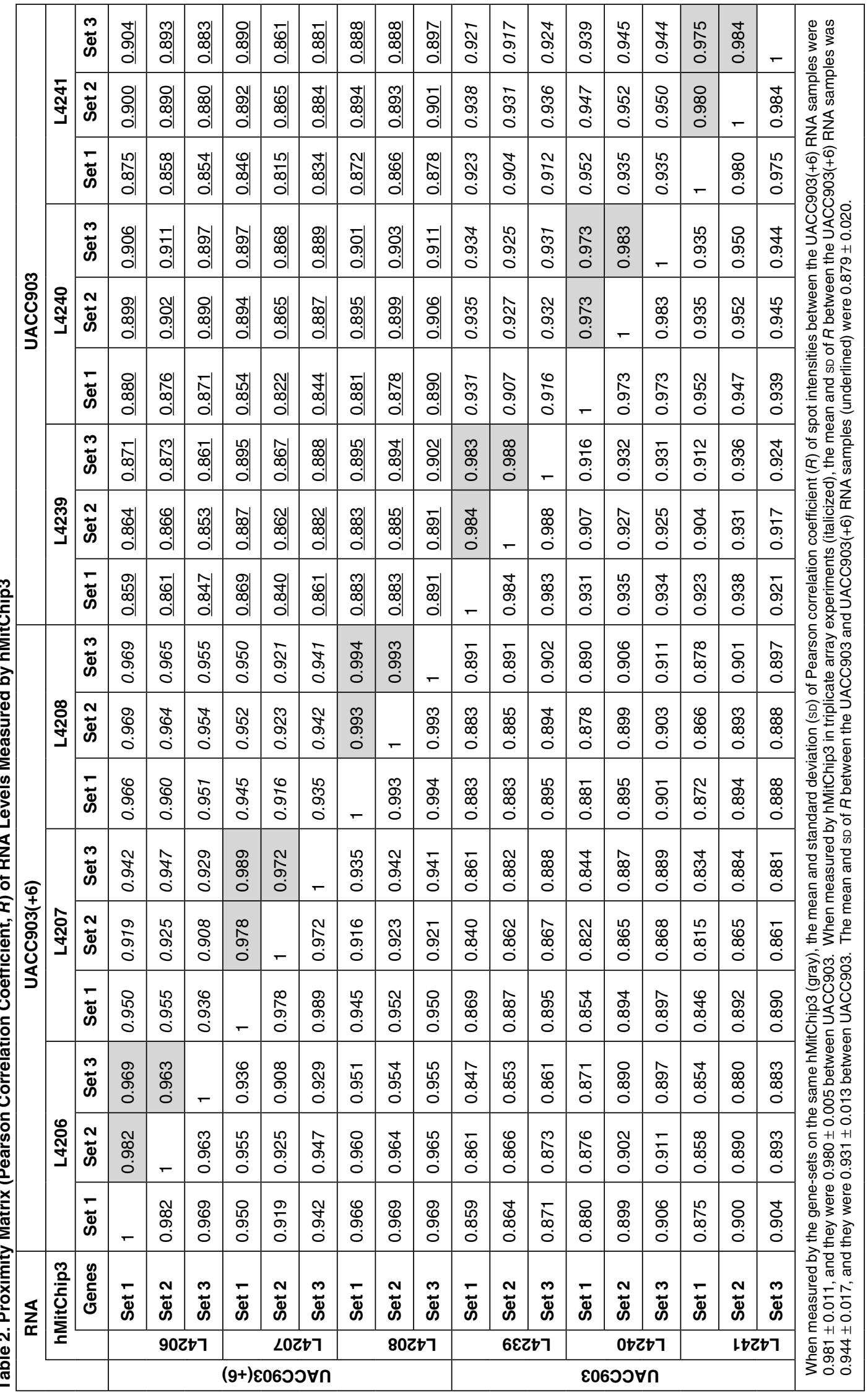




\section{Research Reports}

fluorescent approach, some studies showed evidence for better gene classifiers (37) or accurate expression ratios (38). hMitChip3 as a cDNA microarray can be used with either the one- or two-fluorescent approach according to users' preferences. In this study, the hMitChip3 slides were hybridized with single dye (Cy5)-labeled target DNA from both the test and the reference samples, and the results were validated by quantitative RT-PCR. Two dyes, Cy3- and Cy5-labeling, were tested and validated in experiments conducted with hMitChip2 (14) and remains applicable to hMitChip3.

Microarray data frequently contain a large amount of information on thousands of genes requiring sophisticated bioinformatics tools for appropriate data organization, analysis, and interpretation. The hMitChip3 database, computing procedure, and gene bioinformatics reported herein provide such the tools for rapid identification of candidate genes and biological interpretation of results. The hMitChip3 database structure can be readily modified not only for hMitChip3derived data but also for data generated from other gene chips. The linking of hMitChip3 database to public web sites allows investigators to instantaneously update gene information. Because the output of the array scan is a text file, experienced microarray investigators might select a variety of analytical tools for further data analysis.

The number of candidate genes detected by hMitChip3 is small enough to eliminate the need for high-order analyses such as hierarchical clustering and multidimensional scaling that are based on similarity in gene expression. Instead, a joint mining of differentially expressed genes and biological information within the hMitChip3 database allowed focusing on biological functions relevant to experimental design and interpretation of results. Out of 66 differen- tially expressed genes mapped to specific biological functions, $6 \mathrm{mtDNA}$-encoded genes and 10 nuclear DNA-encoded genes have known functions involved in cell cycle progression and DNA, RNA or protein biosynthesis, and displayed expression patterns consistent with rapid (UACC903) or slow [UACC903(+6)] proliferation status of the cell lines. Although the quantitative RT-PCR verified expression patterns of 6 tested genes, differences indeed exist between microarray and quantitative RT-PCR. In this regard, the differential expression of CCT4 and GABPA detected by quantitative RT-PCR were statistically not significant $(P>0.05)$, in contrast to microarray results. The difference might be partially due to computational difference (577 selected genes used for microarray data normalization versus a single gene $G A P D H$ for quantitative RT-PCR) and partially due to technical difference (higher sensitivity of quantitative RT-PCR than that of microarray).

Table 3. Genes Differentially Expressed between UACC903 and UACC903(+6) Cell Lines

\begin{tabular}{|c|c|c|c|c|c|c|}
\hline \multirow[t]{2}{*}{ Symbol } & \multirow[t]{2}{*}{ Name } & \multirow[t]{2}{*}{ Activity } & \multicolumn{2}{|c|}{ Microarray } & \multicolumn{2}{|c|}{ Quantitative RT-PCR } \\
\hline & & & $903 / 903(+6)$ & $P$ & $903 / 903(+6)$ & $\boldsymbol{P}$ \\
\hline \multicolumn{7}{|c|}{ 1. Cell Cycle Progression } \\
\hline DNMT1 & DNA (cytosine-5-)-methyltransferase 1 & positive effect & 4.36 & 0.000 & 1.69 & 0.029 \\
\hline CCT4 & chaperonin containing TCP1 subunit 4 & positive effect & 2.06 & 0.021 & 1.81 & 0.264 \\
\hline \multicolumn{7}{|l|}{ 3. RNA } \\
\hline GABPA & GA binding protein transcription factor $\alpha$ & transcription factor & 4.07 & 0.001 & 2.03 & 0.263 \\
\hline PPARG & peroxisome proliferative activated receptor $\gamma$ & transcription factor & 0.27 & 0.002 & 0.18 & 0.000 \\
\hline POLR3E & polymerase (RNA) III (DNA directed) polypeptide E & $\begin{array}{l}\text { tRNA, 5S rRNA, U6 } \\
\text { snRNA synthesis }\end{array}$ & 2.73 & 0.007 & ND & \\
\hline HARS & histidyl-tRNA synthetase & histidine activation & 3.56 & 0.002 & ND & \\
\hline SARS2 & seryl-tRNA synthetase 2 & serine activation & 2.33 & 0.011 & ND & \\
\hline \multicolumn{7}{|c|}{ 5. Mitochondria } \\
\hline MTIF3 & mitochondrial translational initiation factor 3 & translation initiation & 3.53 & 0.001 & ND & \\
\hline TUFM & Tu translation elongation factor, mitochondrial & translation elongation & 3.78 & 0.000 & ND & \\
\hline MRPS16 & mitochondrial ribosomal protein S16 & translation & 2.04 & 0.027 & ND & \\
\hline MRPS23 & mitochondrial ribosomal protein $\mathrm{S} 23$ & translation & 5.27 & 0.000 & ND & \\
\hline TRNE & tRNA glutamic acid & glutamic acid carrier & 3.88 & 0.005 & ND & \\
\hline TRNY & tRNA tyrosine & tyrosine carrier & 2.96 & 0.034 & ND & \\
\hline
\end{tabular}

RT-PCR, reverse transcription PCR; TCP1, T-complex protein 1; tRNA, transfer RNA; rRNA, ribosomal RNA; snRNA, small nuclear RNA; ND, not determined. 


\section{Research Reports}

Therefore, we recommend validation of microarray-detected candidate genes by the different method prior to functional study.

Genes involved in cell proliferation were, as expected, up-regulated in a rapidly dividing melanoma cell line (UACC903) in contrast to a slowly dividing derivative cell line [UACC903(+6)]. HDAC3 is the main component of the nucleasome remodeling and deacetylase complex that represses transcription of specific genes with negative control on cell cycle to enhance cell proliferation (39). DNMT1 binds to histone deacetylases and retinoblastoma $(\mathrm{Rb})$ tumor suppressor, leading to loss of functional $\mathrm{Rb}$ (40). CCT4 is required for maturation of cyclin $\mathrm{E}$ and the cell cycle progression from G1 to $\mathrm{S}$ phase (41). NME4 is a nucleoside diphosphate kinase that is involved in RNA or DNA biosynthesis (42). GABPA is the $\alpha$ subunit of GA binding protein that regulates genes involved in cell cycle control, protein biosynthesis, and cellular metabolism (43). In contrast, expression of $P P A R G$, a differentiation-related transcription factor (44), was down-regulated in UACC903 cells compared with UACC903(+6) cells. Genes involved in protein biosynthesis were upregulated in UACC903 in contrast to UACC903(+6) and have yet to be validated. Taken together, the results in this report validate hMitChip3 slides as a measurement tool and hMitChip3 database and computing procedures as an analytic tool, useful to profiling mitochondrial gene expression.

\section{ACKNOWLEDGMENTS}

We thank Drs. Tim McCaffrey and Bi-Dar Wang for critical reviews of the manuscript and Dr. Yinglei Lai for helpful discussion of data normalization algorithms.

\section{COMPETING INTERESTS STATEMENT}

The authors declare no competing interests.

\section{REFERENCES}

1. Scheffler, I.E. 2001. A century of mitochondrial research: achievements and perspectives. Mitochondrion 1:3-31.

2. Margulis, L. 1996. Archaeal-eubacterial mergers in the origin of Eukarya: phylogenetic classification of life. Proc. Natl. Acad. Sci. USA 93:1071-1076.

3. Chinnery, P.F. 2003. Searching for nuclearmitochondrial genes. Trends Genet. 19:6062.

4.Zeviani, M., P. Corona, L. Nijtmans, and V. Tiranti. 1999. Nuclear gene defects in mitochondrial disorders. Ital. J. Neurol. Sci. 20:401-408

5.Leonard, J.V. and A.H. Schapira. 2000. Mitochondrial respiratory chain disorders I: mitochondrial DNA defects. Lancet 355:299304.

6. Enns, G.M. 2003. The contribution of mitochondria to common disorders. Mol. Genet. Metab. 80:11-26.

7. Boudina, S. and E.D. Abel. 2006. Mitochondrial uncoupling: a key contributor to reduced cardiac efficiency in diabetes. Physiology (Bethesda) 21:250-258.

8. Welch, K.M. 1998. Current opinions in headache pathogenesis: introduction and synthesis. Curr. Opin. Neurol. 11:193-197.

9. Wallace, D.C. 2005. A mitochondrial paradigm of metabolic and degenerative diseases, aging, and cancer: a dawn for evolutionary medicine. Annu. Rev. Genet. 39:359-407.

10.Szewczyk, A. and L. Wojtczak. 2002. Mitochondria as a pharmacological target. Pharmacol. Rev. 54:101-127.

11. Dias, N. and C. Bailly. 2005. Drugs targeting mitochondrial functions to control tumor cell growth. Biochem. Pharmacol. 70:1-12.

12. Karunagaran, D., R. Rashmi, and T.R. Kumar. 2005. Induction of apoptosis by curcumin and its implications for cancer therapy. Curr. Cancer Drug Targets 5:117-129.

13. Hu, J., J. Fang, Y. Dong, S.J. Chen, and Z. Chen. 2005. Arsenic in cancer therapy. Anticancer Drugs 16:119-127.

14. Alesci, S., I. Manoli, V.J. Michopoulos, F.M. Brouwers, H. Le, P.W. Gold, M.R. Blackman, O.M. Rennert, et al. 2006. Development of a human mitochondria-focused cDNA microarray (hMitChip) and validation in skeletal muscle cells: implications for pharmaco- and mitogenomics. Pharmacogenomics J. 6:333-342.

15. Manoli, I., H. Le, S. Alesci, K.K. McFann, Y.A. Su, T. Kino, G.P. Chrousos, and M.R. Blackman. 2005. Monoamine oxidase-A is a major target gene for glucocorticoids in human skeletal muscle cells. FASEB J. 19:13591361.

16. Trent, J.M., E.J. Stanbridge, H.L. McBride, E.U. Meese, G. Casey, D.E. Araujo, C.M. Witkowski, and R.B. Nagle. 1990. Tumorigenicity in human melanoma cell lines controlled by introduction of human chromosome 6. Science 247:568-571.

17.Su, Y.A., M.E. Ray, T. Lin, N.E. Seidel, D.M. Bodine, P.S. Meltzer, and J.M. Trent. 1996. Reversion of monochromosome-mediated suppression of tumorigenicity in malignant melanoma by retroviral transduction. Cancer Res. 56:3186-3191.

18. Su, Y.A. and J.M. Trent. 2001. Isolation of tumor suppressor genes in melanoma by cDNA microarray. In B.J. Nickoloff (Ed.), Methods in Molecular Medicine. Humana Press, Totowa.
19. Holland, P.M., R.D. Abramson, R. Watson, and D.H. Gelfand. 1991. Detection of specific polymerase chain reaction product by utilizing the $5^{\prime}-3^{\prime}$ exonuclease activity of Thermus aquaticus DNA polymerase. Proc. Natl. Acad. Sci. USA 88:7276-7280.

20. Bolstad, B.M., R.A. Irizarry, M. Astrand, and T.P. Speed. 2003. A comparison of normalization methods for high density oligonucleotide array data based on variance and bias. Bioinformatics 19:185-193.

21.Hartemink, A.J., D.K. Gifford, T.S. Jaakkola, and R.A. Young. 2001 Maximum-likelihood estimation of optimal scaling factors for expression array mormalization, p. 132-140. In Y. Chen, A.N. Dorsel, E.R. Dougherty, and M.L. Bittner (Eds.), Proc. SPIE, Microarrays: Optical Technologies and Informatics. San Jose, CA.

22. Calvo, S., M. Jain, X. Xie, S.A. Sheth, B. Chang, O.A. Goldberger, A. Spinazzola M. Zeviani, et al. 2006. Systematic identification of human mitochondrial disease genes through integrative genomics. Nat. Genet. 38:576-582.

23. Brandon, M.C., M.T. Lott, K.C. Nguyen, S. Spolim, S.B. Navathe, P. Baldi, and D.C. Wallace. 2005. MITOMAP: a human mitochondrial genome database-2004 update. Nucleic Acids Res. 33:D611-D613.

24. Maitra, A., Y. Cohen, S.E. Gillespie, E. Mambo, N. Fukushima, M.O. Hoque, N. Shah, M. Goggins, et al. 2004. The Human MitoChip: a high-throughput sequencing microarray for mitochondrial mutation detection. Genome Res. 14:812-819.

25. van der Westhuizen, F.H., L.P. van den Heuvel, R. Smeets, J.A. Veltman, R. Pfundt, A.G. van Kessel, B.M. Ursing, and J.A. Smeitink. 2003. Human mitochondrial complex I deficiency: investigating transcriptional responses by microarray. Neuropediatrics 34:14-22.

26.Crimi, M., S.F. O'Hearn, D.C. Wallace, and G.P. Comi. 2005. Molecular research technologies in mitochondrial diseases: the microarray approach. IUBMB Life 57:811818.

27.Alesci, A., Y.A. Su, and G.P. Chrousos. 2004. hMidas and hMitChip: new opportunities in mitochondrial bioinformatics and genomic medicine, p. 329-334. In R.A.S. Long, D.J. Lee, B. Nutter, and M. Zhang (Eds.), Seventeenth IEEE Symposium on ComputerBased Medical Systems. IEEE Computer Society Press. Los Alamitos, CA.

28.Lopez, M.F., B.S. Kristal, E. Chernokalskaya, A. Lazarev, A.I. Shestopalov, A. Bogdanova, and $M$. Robinson. 2000. High-throughput profiling of the mitochondrial proteome using affinity fractionation and automation. Electrophoresis 21:3427-3440

29. Tseng, G.C., M.K. Oh, L. Rohlin, J.C. Liao, and W.H. Wong. 2001. Issues in cDNA microarray analysis: quality filtering, channel normalization, models of variations and assessment of gene effects. Nucleic Acids Res. 29:2549-2557.

30. Shippy, R., T.J. Sendera, R. Lockner, C. Palaniappan, T. Kaysser-Kranich, G. Watts, and J. Alsobrook. 2004. Performance evaluation of commercial short-oligonucleotide microarrays and the impact of noise in making cross-platform correlations. BMC Genomics 5:61. 
31. Barash, Y., E. Dehan, M. Krupsky, W. Franklin, M. Geraci, N. Friedman, and N. Kaminski. 2004. Comparative analysis of algorithms for signal quantitation from oligonucleotide microarrays. Bioinformatics 20:839-846.

32. Wu, W., N. Dave, G.C. Tseng, T. Richards, E.P. Xing, and N. Kaminski. 2005. Comparison of normalization methods for CodeLink Bioarray data. BMC Bioinformatics 6:309.

33.Park, T., S.G. Yi, S.H. Kang, S. Lee, Y.S. Lee, and R. Simon. 2003. Evaluation of normalization methods for microarray data. BMC Bioinformatics 4:33.

34. Wu, W., E.P. Xing, C. Myers, I.S. Mian, and M.J. Bissell. 2005. Evaluation of normalization methods for cDNA microarray data by k-NN classification. BMC Bioinformatics 6:191

35. Churchill, G.A. 2002. Fundamentals of experimental design for cDNA microarrays Nat. Genet. 32(Suppl):490-495.

36. Novoradovskaya, N., M.L. Whitfield, L.S Basehore, A. Novoradovsky, R. Pesich, J. Usary, M. Karaca, W.K. Wong, et al. 2004. Universal Reference RNA as a standard for microarray experiments. BMC Genomics 5:20.

37. Attoor, S., E.R. Dougherty, Y. Chen, M.L. Bittner, and J.M. Trent. 2004. Which is better for cDNA-microarray-based classification: ratios or direct intensities. Bioinformatics 20:2513-2520

38. Peixoto, B.R., R.Z. Vencio, C.M. Egidio, L. Mota-Vieira, S. Verjovski-Almeida, and E.M. Reis. 2006. Evaluation of referencebased two-color methods for measurement of gene expression ratios using spotted cDNA microarrays. BMC Genomics 7:35.

39. Huang, W., D. Tan, X. Wang, S. Han, J. Tan, Y. Zhao, J. Lu, and B. Huang. 2006. Histone deacetylase 3 represses p15(INK4b) and p21(WAF1/cip1) transcription by interacting with Sp1. Biochem. Biophys. Res. Commun. 339:165-171.

40. Robertson, K.D., S. Ait-Si-Ali, T. Yokochi, P.A. Wade, P.L. Jones, and A.P. Wolffe. 2000. DNMT1 forms a complex with $\mathrm{Rb}$, E2F1 and HDAC1 and represses transcription from E2F-responsive promoters. Nat. Genet. 25:338-342.

41. Won, K.A., R.J. Schumacher, G.W. Farr, A.L. Horwich, and S.I. Reed. 1998. Maturation of human cyclin E requires the function of eukaryotic chaperonin CCT. Mol. Cell. Biol. 18:7584-7589.

42. Lacombe, M.L., L. Milon, A. Munier, J.G. Mehus, and D.O. Lambeth. 2000. The human Nm23/nucleoside diphosphate kinases. J. Bioenerg. Biomembr. 32:247-258

43. Rosmarin, A.G., K.K. Resendes, Z. Yang, J.N. McMillan, and S.L. Fleming. 2004. GA-binding protein transcription factor: a review of GABP as an integrator of intracellular signaling and protein-protein interactions. Blood Cells Mol. Dis. 32:143-154.

44. Rosen, E.D., P. Sarraf, A.E. Troy, G. Bradwin, K. Moore, D.S. Milstone, B.M. Spiegelman, and R.M. Mortensen. 1999. PPAR gamma is required for the differentiation of adipose tissue in vivo and in vitro. Mol. Cell 4:611-617.

Received 19 September 2006; accepted 27 November 2006.
Address correspondence to Yan A. Su, Department of Biochemistry and Molecular Biology, GWUSMHS, Ross Hall, Rm. 530, 2300 EYE Street, NW, Washington, DC 20037,USA.e-mail: bcmyas@gwumc.edu

To purchase reprints of this article, contact: Reprints@BioTechniques.com 